\title{
A Conceptual Framework for the Design and Evaluation of Affective Usability in Educational Geosimulation Systems
}

\author{
Elizabeth Furtado ${ }^{1}$, Vasco Furtado ${ }^{2}$, and Eurico Vasconcelos ${ }^{3}$ \\ ${ }^{1}$ University of Fortaleza and of State of Ceara \\ ${ }^{2}$ University of Fortaleza, Mestrado em Informática aplicada \\ ${ }^{3}$ Pontifica Universidade Católica do Rio de Janeiro, Departamento de Informática \\ Av. Washington Soares 1521, Edson Queiroz, Fortaleza, CE, Brazil \\ elizabet@unifor.br, vasco@unifor.br, jfilho@inf.puc-rio.br
}

\begin{abstract}
In this article we propose a conceptual framework for associating the concepts of usability, computer education and affective quality. We analyze the interaction student-teacher under the light of learning strategies used in educational geosimulators for defining the main emotional constructs that are involved in this process. We elaborate this initial analysis by identifying which interactive objects should be associated with the identified emotional constructs. We associate these objects with an architecture that defines the basics components of an educational geosimulation system as well as the learning strategies used in this context. We illustrate the utility of the framework with an evaluation of an education geosimulator for police training as well as an evaluation of the student's satisfaction during the interaction in different scenarios.
\end{abstract}

Keywords: Affective emotional quality, Educational System Evaluation.

\section{Introduction}

Intelligent Tutoring Systems (ITS) have been studied since the 1970s and an increasing variety of tools have emerged since then, leveraging diverse techniques from different areas of computer science and particularly from Artificial Intelligence. Educational agent-based simulation systems are one of these, in which intelligent agents support the interaction between the simulation model and the user [10]. One of the recent advances in these systems was the combined use of Geographical Information Systems (GIS) with multiagents for simulation of social or urban environments, which characterizes geosimulation [1]. With the computational development of GIS, bringing precision and realism to simulation [25], educational tools are also benefited with richer human-computer interaction strategies.

Despite the aforementioned advances, few studies have paid attention to the usability aspects of the educational systems that involve complex phenomena in urban centers, where the interaction with GIS is intense. These aspects must be taken into account in the systems design phase and can also be useful for evaluating the final quality of the User Interfaces (UIs). Moreover the approaches that focus on the 
usability of educational systems concern only the effectiveness of the user-system interaction (the number of successful task completions) and the efficiency (the time required to complete an interactive task) [16].

In this article we follow a multidisciplinary approach by proposing a conceptual framework for associating the concepts of usability, computer education and affective quality. Our work goes beyond the traditional view of usability by considering the affective dimension of the UI-a topic that has gained ground in recent HCI research. We claim that the affective aspect (such as users' feeling states and their involvement with the content) is particularly relevant in the context of educational systems, since learning depends strongly on how synergetic the relationship is between teacher and student. The framework construction is done in two steps. First, we analyze the interaction student-teacher under the light of learning strategies used in educational geosimulators for defining the main emotional constructs that are involved in this process. From this initial analysis we evolve the framework by identifying which interactive instruments should be associated with the identified emotional constructs.

We have chosen to associate the affective usability aspects with an architecture proposed in [7] that defines the basic components of an educational geosimulation system as well as the learning strategies used in this context. We then provide designers with orientation of how to take these aspects into account already in the interaction design phase of these systems. Moreover, we consider that our framework is also appropriate to evaluate the users' satisfaction that refers to the affective quality they have about the system in interaction. In the final part of the paper we present how the conceptual framework can be used to evaluate an already deployed system for training police officers. Finally, we evaluate the user's satisfaction using this system during the realization of different scenarios of a training process.

\section{Affective Quality in Interactive Systems: Design and Evaluation Issues}

HCI has often attracted considerable attention from academic communities by integrating these concepts into an educational system development process in order to obtain more usable systems. Traditionally, the usability of a developed system has been evaluated to assure both its effectiveness (such as the number of successful task completions) and efficiency (such as the time required to complete an interactive task). Recently, these assumptions have been revisited and broadened to embed the concepts of the affective quality theories. Affective quality is related to the user's emotional responses (such as the affect, activity and attitude of the users) in regards to the system that they are experiencing [3]. For instance, a learning system may elicit enjoyment (e.g. pleasure). Then, the users may continue using it for a long time and become emotionally absorbed (e.g. engagement by a content that matches with their objects of interest, preferences and restrictions). Finally, the users may decide that they like a specific learning task, which leads to the formation of an attitude (e.g., a summary evaluation of an experience supported by explanations).

In [19], the author investigates the role of emotions in the design and evaluation of a UI for any interactive product in three levels of the human brain mechanism: on the visceral level, the emotional reactions are associated to the nature of the UI (aesthetics 
and beauty); on the behavioral level, the emotional reactions are associated to the use of the UI (effectiveness, efficiency and errors). On this level, Norman situates the traditional usability notion; and on the reflective level, the emotional reactions refer to the meaning of the UI (rationalization and intellectualization of a product). Table 1 depicts the relation existing among the usability and affective quality concepts in which must be both considered when evaluating the users' satisfaction (such as their subjective perceived ease of use) of learning systems.

Table 1. HCI concepts from usability engineering and affective disciplines

\begin{tabular}{ll}
\hline USABILITY & AFFECTIVE QUALITY \\
\hline Focus on interactive task design & $\begin{array}{l}\text { Focus on emotional design, } \\
\text { captology, funology }\end{array}$ \\
$\begin{array}{ll}\text { Concepts of usability: task, effectiveness, } \\
\text { efficiency and errors }\end{array}$ & $\begin{array}{l}\text { Concepts of affective quality: feeling } \\
\text { states as pleasure in interactive experi- } \\
\text { ences }\end{array}$ \\
$\begin{array}{ll}\text { Activity of users based on task execution, } \\
\text { their preferences and restrictions }\end{array}$ & $\begin{array}{l}\text { ation, context and participation. } \\
\text { ration }\end{array}$ \\
$\begin{array}{l}\text { Attitude of the users supported by task } \\
\text { completion and representation of interac- } \\
\text { tive objects associated to the tasks }\end{array}$ & $\begin{array}{l}\text { assistances through persuasive and } \\
\text { user difficult strategies }\end{array}$ \\
\hline
\end{tabular}

The emphasis on both of these concepts entails a complete evaluation of the users' satisfaction as for: 1) their ability to learn about the system and to use it in a creative way. This ability that the users must have is the direct reflex of their comprehension of the UI, specifically, of the representations of its interactive objects [22]. A possible question to pose to the users is the following: How many of the interactive objects representations were not associated with your intention of use? and; 2) their emotional responses (feeling states, trust, engagement) with the system. Some evaluation methods have included the following questions in users' satisfaction tests: how fun was the user interface? How interesting was the content presented? How persuasive was the user interface? It is worthy mentioning that this broader notion of usability requires a user-centered design process, in which users must be analyzed by the designers in order to build better interactive objects (as widgets) and to understand the emotional effects that Interactive Systems (ISs) will have on users [4].

\section{Educational Geosimulation Systems}

Simulation aims to represent one phenomenon via another. In educational terms, simulation is important because it allows learning through the possibility of doing [21]. On the other hand, Social or urban environments are dynamic, non-linear, and made of a great number of interacting entities, characterizing a complex system. The use of Multiagent System to simulate social environments has become broadly used [2] [13].

Recently [8] brought together the components of what they consider relevant for the development of urban activities training by means of simulation systems and proposed the Educational Geosimulation Architecture (EGA). EGA encompasses the 
basics components of an educational geosimulation system serving as a basis to developers of this type of system. EGA follows a traditional architecture of an ITS in which three main models are distinguished: the student model, the teacher model and the domain model. However, some particular aspects are present in EGA. Geosimulators are used as a tool of the teacher model because the topic of study is dynamic and the practice in reality involves high risks and costs. The use of a GIS allows for the appropriate representation of the simulation environment (domain model). The multiagent simulation approach is followed because it provides an appropriate computational representation of independent entities that interrelate within the same environment. In this educational context, a multiagent simulation platform contains two types of agents: domain agents, that represent the domain model and/or student model and pedagogical agents representing the teacher model. Last but not least important, there are the user interfaces that are the communication channel between the system and the student. Figure 1 depicts the previously mentioned EGAs main components and indicates the three basic strategies of learning that it entails. The more traditional strategy is learning by instruction that is obtained from the material (information, examples, concepts) provided by the teacher. By using the simulation the student learns by doing. The assistances provided by the teacher to help the better understanding of the phenomena underlying the simulation leads to a process of learning by reflection.

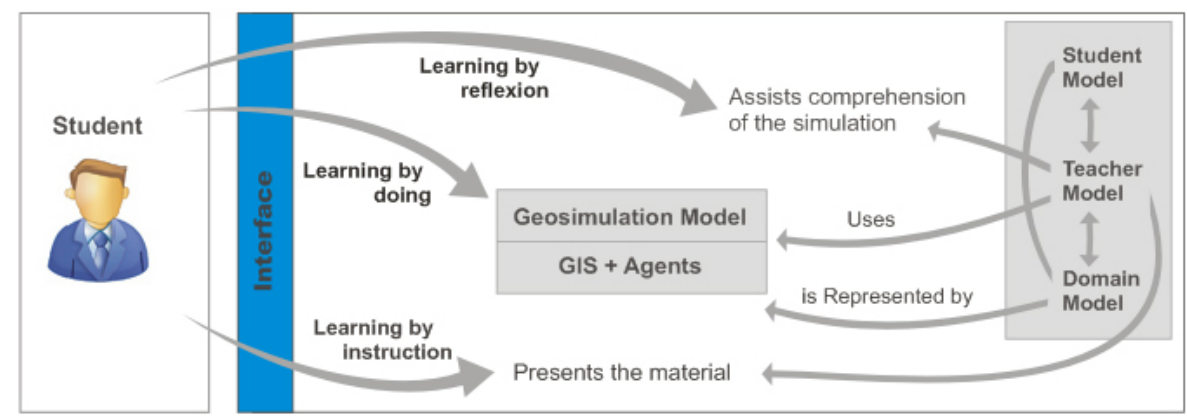

Fig. 1. Interaction among the Educational Geosimulation Architecture Components

\section{Affective Usability Aspects in Educational Geosimulation Systems}

We draw from this work the focus on some HCI concepts describing a multidisciplinary strategy of generating a conceptual framework from education, user interaction and affective quality theories (see Table 2). From the EGAs learning strategies, we analyzed the possible emotional responses of affect, activity and attitude that the students can have in the interaction process with the educational system. We considered, for instance, that the affect (such as feeling states) is a neurophysical state that is consciously accessible as a simple, non-reflective feeling [26]. This is the reason we associated it to the learning by instruction strategy. In scenarios of this type, the student is unconsciously accepting the instruction as being the truth 
(indicating a high level of trust in the teacher, or even of submission). The simulation and explanation strategies, in turn, raise emotional responses related to the behavioral (activity) and reflective (attitude) levels of a person, respectively. Then we associate affective usability design aspects that might elicit positive emotional responses from the students. For instance, an instruction-based system can be designed with sophisticated technological resources (as tangible interfaces), that positively influence users' experience on an emotional level (as having fun) when interacting with it. Note that a same system can elicit a combination of emotions. For instance, a system can be viscerally attractive through its UI and elicit reflection through the way explanation is displayed.

Table 2. Affective usability aspects in educational geosimulation systems

\begin{tabular}{|c|c|c|}
\hline Learning Strategies & Emotional Responses & $\begin{array}{l}\text { Affective Usability } \text { Design } \\
\text { Aspects }\end{array}$ \\
\hline $\begin{array}{l}\text { Learning by in- } \\
\text { struction }\end{array}$ & $\begin{array}{l}\text { Affect } \\
\text { Feeling states (motivated, enthusias- } \\
\text { tic, calm) for using a system that is } \\
\text { beautiful, attractive and with less } \\
\text { constraining interaction; Pleasure in } \\
\text { having fun. }\end{array}$ & $\begin{array}{l}\text { Representation of interactive } \\
\text { tasks through interactive } \\
\text { agents; } \\
\text { Manipulation and animation of } \\
\text { interactive objects. Look, } \\
\text { sound and feel dominate. }\end{array}$ \\
\hline $\begin{array}{l}\text { Learning by doing } \\
\text { (simulation) }\end{array}$ & $\begin{array}{l}\text { Activity } \\
\text { Engagement in figuring simulations } \\
\text { out (perceived ease of use, no feel- } \\
\text { ing of risk and failure); Involvement } \\
\text { in understanding the simulation } \\
\text { content; Trust }\end{array}$ & $\begin{array}{l}\text { Support to trials through his- } \\
\text { toric of simulations; } \\
\text { Contextual and participatory } \\
\text { simulations }\end{array}$ \\
\hline $\begin{array}{lr}\text { Learning by reflec- } \\
\text { tion } & \text { (Thinking } \\
\text { through } & \text { assis- } \\
\text { tances) } & \end{array}$ & $\begin{array}{l}\text { Attitude } \\
\text { After a superficial involvement, a } \\
\text { sense of true commitment in under- } \\
\text { standing the simulation context, that } \\
\text { can bring to the formation of atti- } \\
\text { tude. }\end{array}$ & $\begin{array}{l}\text { Persuasive techniques and } \\
\text { difficult-regulation strategies } \\
\text { for providing adaptive, helpful, } \\
\text { and structured assistances. }\end{array}$ \\
\hline
\end{tabular}

The aspects illustrated in Table 2 are admittedly incomplete: they are meant only to spawn new ways of thinking about the users' satisfaction with these systems by not only focusing on the systems' efficiency. The aspects do not address, for instance, characteristics of context in which the interaction should take place. They refer more to the way in which the interactive objects that represent the learning strategies should be perceived by the users when manipulating, creating, visualizing or controlling these objects in their learning experiences. Our goal is to provide design concerns elucidated from theoretical literature when appropriate, which can help professionals both to design and to evaluate these kind of educational systems. Specifically, we intend to show how attention paid to users' emotional responses can be used as a basis for developing and evaluating these systems in order to elicit positive responses from students. The affective usability design aspects were classified into three groups as follows. 


\subsection{Eliciting Emotional Responses Through the Representation of the Interactive Elements}

Users expect to have not only appropriate functionality and usability, but pleasure ("benefits") in using the UI as well [12]. For this reason, many high-tech design solutions are being defined as patterns, in order to be reused in other situations. Interaction Patterns represent best design solutions for known interaction problems [23]. As the interactive agent notion is usually used in educational systems, it can be modeled as an interaction pattern and be characterized by having an interactive representation. For instance, an agent can be of the anthropomorphic agent pattern type, when realized through synthetic characters in the UI of affective systems. The description of a pattern should include the possible constraints involved in the interaction. For instance the designer should know that anthropomorphic effects can cause interaction problems when considering the fact the user can expect the system to be intelligent and cognitively potent. This expectation may lead to frustration in the user when the system can not meet these expectations [11].

In a GIS, the graphical representation presented to the user through the UI is the result of interactive objects which represent agents, users' action and/or context of study. Some aspects in the UIs of the GIS can contribute to enhance the users' satisfaction for different reasons, in particular:

- Direct Manipulation. Users need to have complete control over the system, it can be hard to accept characters in the interface that run outside their control. Users got irritated and frustrated when they could not figure it out [11]. It is important to provide users with support for free exploration when manipulating a map. The idea is to allow, for instance, the user to query the object about its features or even to control its behavior in a certain situation;

- Animation: animation through several effects (such as color changes, panning, and dynamic links) can make a UI more memorable and vivid, and more enjoyable to use, leading users to have fun. In agent-based systems, agents can be designed to navigate (virtual) spaces with movement patterns to simulating urban population as collectives of individuals with associated behaviors and traits.

We cannot forget the studies coming from other fields (such as semiotic, ergonomic, communication), related to different aspects that can bring aesthetic and beauty to the system, and consequently pleasure to the users [26]. In semiotic design; studies are about the choice of significant symbols that are an abstraction of the object of the real world and the definition of how these symbols are used during the communication of the users with the system [22]. In communicational, ergonomic and graphical design, studies are in many styles in a variety of colors in order for designers to create the artistic UI design.

\subsection{Eliciting the Emotional Responses in Simulation Experiences}

In a simulation process, the possible users' emotional responses can be elicited from:

- Their engagement in figuring simulations out. The design aspect refers to the support an educational system gives to trials through historic of simulations, free exploration, and treatment of errors. For instance, if the users can make 
trials as many times as they want, with no feeling of risk and failure, then they will have more intentions to do so. The number of times the users figured simulations out and the time spent in this process are important criteria to measure their engagement in interactive simulation experiences.

- Their involvement in understanding the simulation content (as the results, which can be boring/interesting). The design aspects refer to the simulation content's characteristics, which can be contextual. It means that the simulation takes place on a map that represents the context of the displayed content (the agents). In learning theories, it is known that users are more involved with the content when it represents objects of their own interest. For instance, if real world scale map is used, the users will be more involved in the simulation context. Such closeness with their real object makes the UI more comprehensible to the user. Another important factor of HCI to elicit pleasure of users in learning experiences is to allow them participating as active subject in the simulation process [18].

\subsection{Eliciting the Emotional Responses in Assistance Experiences}

Like teachers, educational systems can provide students with different modalities of assistances, such as: a) Explanation, which refers to the act or process of explaining something; b) Hint, which is a brief or indirect suggestion, a tip; and c) tutorial, this refers to instructions describing how the users can proceed at a certain moment.

Like teachers, these systems should motivate students to try out one or more assistances. Some systems' characteristics are important to encourage users in this decision moment (of looking for assistance or not) such as: trust and credibility in assistances to obtain. Several user studies show that transparency is a key factor to trusting learning systems. In [17], some explaining techniques show users the provenance of the explanations. Having decided to get help, the users start looking for interesting assistances around the screens by visiting pages, navigating among menu items, reading the main information, and so on. In this superficial involvement, persuasive interactive techniques can influence users to stay active and loyal in assistance and entail in a profound reflective process. It is expected that the understanding of the obtained assistance makes users change their attitudes.

Persuasive technologies, studied in the area of Captology, can interactively manipulate what people think and do. Examples of these technologies are the following: persuading through customization, simplifying and guided persuasion [6]. This same author gives an example that customized explanation provides users with tailored information to achieve a persuasive result. To encourage users to take action against polluting organizations, a customized graphical explanation could be given through a map when users enter their zip code. The map can enable users to view the location of pollution sources relative to where they live, work, or attend school. Tailored information is more effective than generic information in changing users' attitudes and behaviors. Hint is a kind of simplifying persuasive technique, because users know they will find brief help. Tutorial is a guided persuasion technique, and it provides opportunities to persuade users along the way. Another way to motivate students to get the assistances refers to providing them with appropriate levels of assistance regardless of students' skill. Many works refer to the difficulties in dealing with the 
conflicting goals of expanding system functionality (as more elaborated assistances) for expert users while simultaneously keeping the system easy to use for novice users. In [14], Larson specified difficult-regulation strategies which can be useful in computer games, such as: a) user skill selection, when the users have the power to choose what level of game play they want; and b) explicit and implicit stage progression: in implicit staging, game play becomes more difficult the longer the users play; in explicit staging, when the users complete a stage the game may stop to congratulate them, and the users start the next stage. In this educational context, explicit assistances can also be given in function of the historic of simulations.

\section{Analyzing the Student's Satisfaction in Learning Experiences}

In this section, we describe an educational geosimulator that follows EGA architecture and show how the framework previously define can be used to evaluate the users' satisfaction from an emotional perspective while using this system.

\subsection{Case Study: The ExpertCop System}

In brief, the ExpertCop system supports learning by means of simulation of phenomena that provoke crime in an urban area. Initially the student provides the system with a police resource allocation plan. Then the system simulates crime for a predefined period. The goal is to lead the students to understand the consequences of their allocation as well as understand the cause-and-effect relations. In ExpertCop, the simulations occur in a learning environment and along with graphical visualizations that aid the users' learning. The domain agents are the police team, the criminals and the targets (notable points). Criminals are the most important agents in the simulation process. Their behavior is based on a rule base which orients them to look for targets and to commit crimes (see [8] for a detailed description). The Pedagogical Agent (PA) represents the teacher model and aims at helping the users to understand the phenomena represented by the simulation.

The interaction with the domain agents is done at two moments. First, before the simulation, the user must allocate the police in the areas to be patrolled and available on the geoprocessed map. Figure 2 shows the interface for this allocation process and describes its main functionalities. Crimes are represented on the map as points-in red if the crime is committed and in green if it is not. The goal of the user is to provide a good allocation, which prevents the occurrence of crimes to the greatest extent. Second, during the simulation the movement of the police patrol routes is shown. The user can follow the simulation process in the simulation interface. At the end of the simulation process, the user accesses the system's pedagogical tools. Upon each new allocation performed, the system can comparatively evaluate the simulated moments, showing the user whether the modification brought a better effect to the crime rate or not. After the simulation, interaction is possible via queries to crimes that occurred. It is up to the PA to answer these queries. 


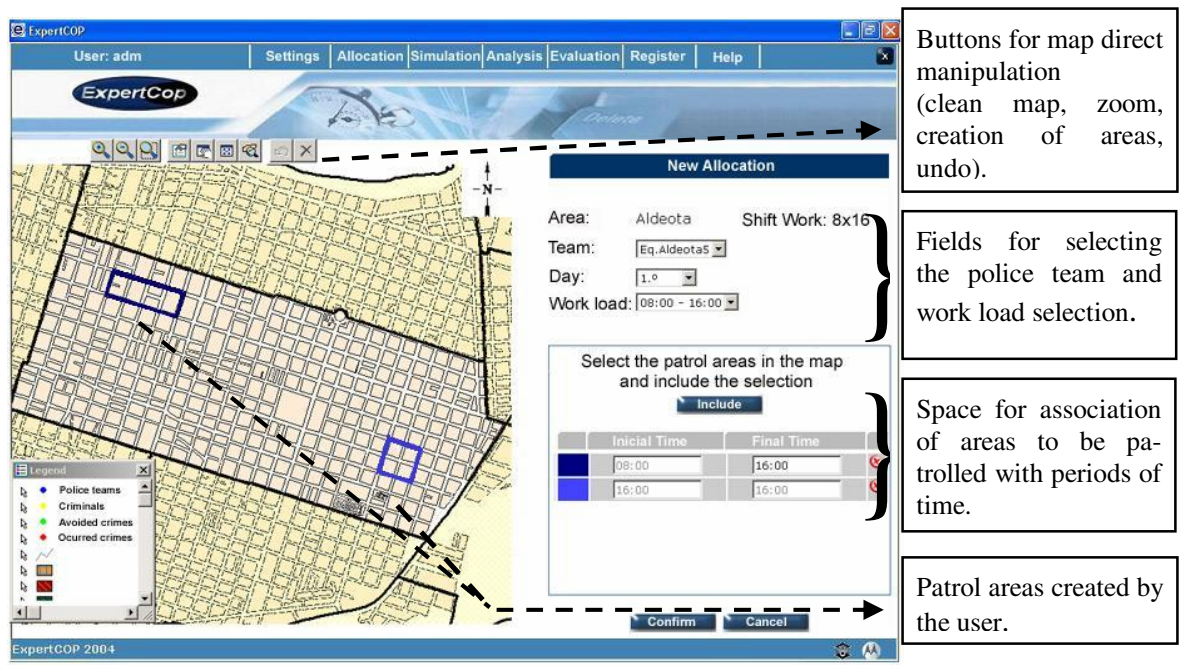

Fig. 2. ExpertCop's police allocation interface

With relation to the interaction with the PA, it is supposed that the PA elicits users' pleasure in learning experiences and a formation of attitude by providing them with different assistances. The PA uses two distinct forms to explain the events of the system, the explanation at a micro-level and at a macro-level. At a micro-level, to explain the simulation events (crimes), the system uses a tree of proofs describing the steps of reasoning of the criminal agent responsible for the event. This tree is generated from the process of the agent's decision making. The agent's evaluation of a crime is represented by a set of production rules. At a macro-level, for the explanation of the system's emerging behavior, the PA tries to identify patterns of the crimes generated in the simulation (see [7] for details of how this is done). The concepts learned by the system are displayed to the user as hints of the following type: "Did you realize that crime: theft, victim: vehicle, week day: Saturday, period: night, local: residential street, neighborhood: Aldeota Beach frequently occur together?" Having this kind of information, the user can reflect on changes in the allocation, aiming to avoid this situation. Figure 3 shows the functionalit ies for visualization.

\subsection{Analyzing the Emotional Aspect of the Students Using ExpertCop}

ExpertCop was used to support a course at the Ministry of Justice and the National Secretariat of Public Safety. ExpertCop was intended to help police officers reflect on the forms of treatment and analysis of information and how these influence the understanding of crime. The audience was made up of thirty professionals in the area of public safety: civil police officers, chiefs of police, and military police (which are the majority). A quantitative analysis of the effectiveness of ExpertCop in the learning process is discussed in [8]. In this paper, we will concentrate on the description of an empirical qualitative analysis of the users' satisfaction, in the light of the aforementioned concepts. 


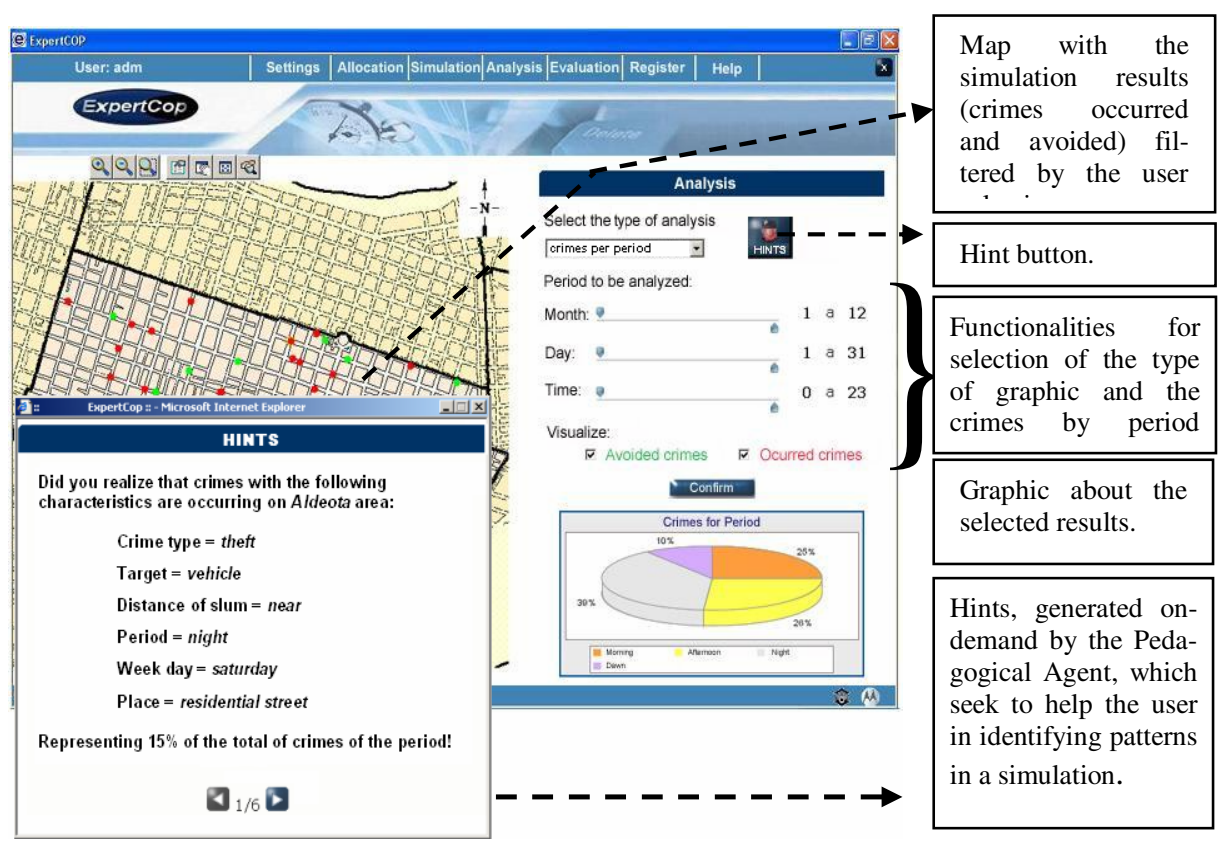

Fig. 3. Visualization of the Assistances

The testing session was composed by the following scenarios of the training process: familiarization, resource allocation, simulation and evaluation of simulation results. It took place from 30 to 40 minutes. Initially, students made use of the tool in an illustrative simulation to familiarize themselves with the functionalities. In the resource allocation scenario, training was carried out by a set of at least two simulations in city areas. In the first simulation, the students had to create and configure a certain number of teams (according to the size of the area), allocate them on the map, and activate the simulation. At the end of the first simulation we asked the students to identify, according to their beliefs, factors (concepts) that influenced the occurrence of the crimes. They did so by observing the map of the crimes that occurred and those that were avoided. They focused on geographical and/or visual factors that directly effect the crime rates. After collecting the students' concepts, we allowed them to use the pedagogical support of the system (hints, explanations and evaluations). After this moment, the collection of factors influencing the crimes was carried out again. In the subsequent simulation, we repeated the same area to serve as a comparison with the initial simulation already completed, and allowed them to make their allocations and use the pedagogical support of the tool according to their needs. At the end of the process we also apply a questionnaire asking about how fun the system was and how difficult to learn its use was. We participated of the testing session as experimenters. A discussion about the users' emotional responses evoked by the system is done as follows.

Arousal. The first reaction of the most part of the users (in case the students) during the initial contact with the system was of complete pleasure. Some of them asked to 
have a license because they wanted to use it as a tool of decision-making support. This emotional response of the users is associated to the Wow factor of the visceral level: wow, I like it. I want it [19]. The aesthetic aspect of the interface with interactive object and colors well chosen, and the fun aspect of the game-like structure are potential responsible for this.

Pleasure of fun vs. quality of learning. Being fun is particularly delicate in gamebased ITS systems. Sometimes the system interface is so fun that it leads the user to game with the system without any intention of learning the process behind the game. It is important, then, to design the ITS to be resistant to the game factor, i.e. the user obtains good scores in the game by just guessing. Moreover, the game aspect cannot bypass the main goal which is learning from the use of the system. The difficulty of having good performance (in terms of crime rate reduction) just guessing is a particular aspect of ExpertCop and was analyzed in detail in [8]. Moreover, the sequence of the scenarios that consists of a phase of analysis of the simulation results is also an important factor because it leads the user trying to understand the simulation model.

Pleasure of using. Several graphical characteristics elicit from the users good feelings leading them to use comfortably the system. Assistances are provided in several ways, and are associated with the agents allocated on the map. One of these assistances is the possibility of visualization on map of notable points such as squares, gas stations, drugstores, schools, etc. Doing so, the user can allocate the police agents by directly drawing on the map the routes that must be followed by these agents while taking into account the hot spots to be monitored. Even the task of police allocation, that our observations and answers of the questionnaire have captured as being the most difficult to do in the system, was considered agreeable to do. Our analysis is that the direct manipulation of the agents representing the police officer in the map is the main factor that influenced this feeling.

Involvement. All the users found the results of the simulation very interesting, in particular, six users who lived in the same city represented in the GIS. They were eager to plunge into allocation then anxious to see the results. They likely took far longer than the others for performing the allocation process. These emotional responses reflected on an emotional involvement of the users with the content. We think it was provoked by the contextual identification aspect of the users with their area of work. Moreover, the simulations can be restarted as many times as the student wants. The process is friendly and a historic of simulation results can be accessed allowing the users to follow their performance.

Sense of persuasion. Users reacted most positively to the hint explanation (showing crime patterns) that made them reflect on their allocation, commenting that they understood why some crimes have not been avoided. Remember that the hints are shown to the users as a pop up window and that they can concomitantly verify their validity while visualizing the patterns in the map. The idea herein contained is to influence the users passing through the UI (in this case, through the hint button) messages that could affect the users' intentions of use (in other words, to motivate them to learn by exploring the assistances). This is a good example of how the interface, by simultaneously contemplating distinct modalities, has enhanced the way information was provided to the users and consequently persuading them. 
Trust. In the first simulation users trusted in the system accuracy but not in its results. They trusted more in the system, when the explanation of the occurrence of the events was given. They could interpret the model behind the simulations. A missing point that would add credibility to the system is a summary about the origin of the information used in the simulation and makes it available in the help option in the main menu of the tool.

Formation of attitude. As for the users' attitude to the system, it is supported by explanations about the criminals' behavior for a certain scenario of preventive police that they defined. Crimes are shown on the map in terms of red dots. The user can click on these dots emulating a query of the type "why have you committed this particular crime?" Using concepts familiar to the users' discourse domain, rule-based explanation emulates the criminal's answers to the users' query. This interactivity process reinforces the users' attitude towards the system because of its contextual feature and persuasiveness.

Participation and Collaboration. During the iterative process of allocationsimulation-visualization of results, users applied some spontaneous collaborative practices (such as: users wanted to make comparisons among their simulation results and/or to comment about their allocation proposals) to identify similar strategies among themselves. They usually collaborated with the colleagues beside them and/or with a user on another computer. The possibility to accompany their evolution in the simulations by means of bar graphs helped this participation process. However, we consider that the lack of tools for collaboration is a shortcoming because would allow collaborative problem solving enriching the learning and the user involvement. In addition, a participatory simulation in which the student participates of the simulation process (reallocation police routes, for instance) would bring more realism to the system as well as more engagement of the student.

\section{Related Works}

There are some works that make the relation between affect and usability in several domains and in different phases of a system life-cycle. In the interaction design phase, Höök [141] suggests first understanding the users' emotional states with experiences while using the technology in the early development phases of a system when understanding their needs. In the evaluation phase, De Angeli and her colleagues [5] showed that usability and aesthetic quality criteria influence users' preferences in relation to two different interface styles (traditional menu-based and interactive animated metaphors). As we, Chorianopoulos \& Spinellis [3] elaborated a conceptual framework based on Norman's affect model. This framework was used to evaluate interactive applications for Digital Television and presents a clear separation among the UI and the TV content. In this work we followed a different approach considering the system as a whole. As we can not measure a system independently, we preferred to associate these concepts by integrating them to the generic learning strategies. Moreover Zhang \& Li [26] made references to several works that associate design features to affective constructs in different domains, but there was not any mention to works in the educational domain. In learning systems, the association of these concepts is still an open issue. In [20], an affective model is included in tutorial system 
architecture. Their idea is to identify the students' emotional status for providing adaptive assistances. Affective relations of power and identity among students have been explored in collaborative virtual learning systems, but there is no relation between these concepts and the usability in these systems [16]. Several works in games and entertainment [9] [15] use simulation with an educational propose. Game simulators have a different pedagogical strategy, because they focus on the results of the simulation emphasizing only the fun aspect of the interface. In learning systems the most important aspect is the process itself, and it should also include formation of attitude.

\section{Conclusion}

We identified several characteristics in the interactive learning processes that raise students' emotional responses according to the Norman's affect model. Then we proposed a conceptual framework for affective usability that should be considered in a user-centered design and/or in the evaluation of educational geosimulation systems. As a case study and with the goal to illustrate how the framework can be used, we evaluated an educational system deployed for the area of law enforcement by analyzing the users' satisfaction regarding their emotional responses in interactive learning situations. For this purpose, training courses with police officers interacting with the system were observed and three different scenarios were taken into account to discuss the results. The perceived behavior of students in ExpertCop revealed the association of their satisfaction in using the system with the HCI concepts described in our framework. We also describe the role that the interfaces played in the system to evoke positive emotional responses from the students, namely the capability to involve them with the content, the feeling of fun, arousal, etc. Such a description also contributes to designers who are going to create new software because it exemplifies how the aspects considered in the framework were used. An important point for future research consists of investigating how important the difference between traditional learning simulation and educational geosimulation is. Our feeling, in which requires validation, is that needs to be validated by comparison is that geosimulation provokes more emotions due to the proximity of reality aspect that is obtained from map using.

Acknowledgement. The first author thanks CAPES grant 2765055 for the financial support.

\section{References}

1. Benenson, I., Torrens, P.M.: Geosimulation: object-based modeling of urban phenomena. Computers, Environment and Urban Systems. Forthcoming (2004)

2. Billari, C.F., Prskawetz, A.: Agent-Based Computational Demography: Using Simulation to Improve Our Understanding of Demographic Behaviour. Phisica-Verlag, Germany (2003)

3. Chorianopoulos, K., Spinellis, D.: user Interface Evaluation of interactive TV: a media studies perspective. Univ. Access Inf Soc. 5, 209-218 (2006)

4. Cooper, Reimann: The Essentials of Interaction Design. Wiley, Chichester (2003) 
5. De Angeli, A., Sutcliffe, A., Hartman, J.: Interaction, Usability and Aesthetics: What influences users' preferences? DIS (2006)

6. Fogg, B.J.: Persuasive Technology. Morgan Kaufmann, San Francisco (2003)

7. Vasconcelos, E., Furtado, V.: Educational Geosimulation. In: Brasileiro, F., Mendes, M. (eds.) Advances in Computer-Supported Learning, Idea Group (2006)

8. Furtado, V., Vasconcelos, E.: Geosimulation in education: a system for teaching police resource allocation. Intl. Journal of Artificial Intelligence in Education 17 (2007)

9. Galvão, J.R., Martins, P.G., Gomes, M.R.: Modeling Reality with Simulation Games for a Cooperative Learning. In: Winter Simulation Conference, pp. 1692-1698. Orlando, FL (2000)

10. Gibbons, A.S., Lawless, K.A., Anderson, T.A., Duffin, J.: The web and model-centered instruction. In: Khan, B.H. (ed.) Web-based training, pp. 137-146 (2001)

11. Höök, K.: User-Centred Design and Evaluation of Affective Interfaces, In: Ruttkay, Zs., Pelachaud, C. (eds.) Kluwer (2004)

12. Jordan, P.: Designing Pleasure Products. Taylor \& Francis (2002)

13. Khuwaja, R., Desmarais, M., Cheng, R.: Intelligent Guide: Combining User Knowledge Assessment with pedagogical Guidance. In: Lesgold, A., Frasson, C., Gauthier, G. (eds.) ITS 1996. LNCS, vol. 1086, Springer, Heidelberg (1996)

14. Larson, J.: Out of the video arcade, into the office: where computer games can lead productivity software. Interactions ACM. vol. XIV.1 (January 2007)

15. Leemkuil, H.H., Jong, T., de Hoog, R., de Christoph, N.: KM Quest: a collaborative internet-based simulation game. Simulation \& gaming 34, 89-111 (2003)

16. Mattos, F.L.: Concepção e desenvolvimento de uma abordagem pedagógica para processos colaborativos a distancia utilizando a internet. PhD. UFC (2005)

17. Mcguinness, D., Li Ding, Furtado, V., Glass, A., Zeng, H., Chang, C.: Explanation Interfaces for the Semantic Web: Issues and Models. In: Proc. of SWUI., vol. 1 (2006)

18. Miller, C.: Digital Storytelling: a creator's guide of interactive entertainment. Elsevier, Amsterdam (2004)

19. Norman, D.: Emotional Design. Basic Books (2004)

20. Perez, Y., Gamboa, R., Ibarra, O.: Modeling Affective Responses in Intelligent Tutoring Systems. In: Proc. of IEEE ICALT, IEEE Computer Society Press, Los Alamitos (2004)

21. Piaget, J.: Le comportement, moteur de l'évolution. Gallimard (ed.) Paris (1976)

22. Souza, C., Uma, S.: Abordagem Semiótica na Utilização dos Recursos Visuais em Linguagens de Interface. Isa Haro Martin (2003)

23. Sousa, K., Mendonca, H., Furtado, E.: Applying a Multi-Criteria Approach for the Selection of Usability Patterns in the Development of DTV Applications. In: IHC' 2006 (2006)

24. Stephanidis, C.: User Interfaces for All. In: Stephanidis, C. (ed.) User Interfaces for All Concepts, Methods, and Tools, pp. 3-17. LEA, Inc., Mahwah, NJ (2001)

25. Wu, F.: Complexity and urban simulation: towards a computational laboratory. Geography Research Forum 22, 22-40 (2002)

26. Zhang, P., Li, N.: The Importance of Affective Quality. In: CACM'04 (2004) 